\title{
Mating-Induced c-fos Expression Patterns Complement and Supplement Observations after Lesions in the Male Syrian Hamster Brain $^{a}$
}

\author{
SARAH WINANS NEWMAN, ${ }^{b, d}$ DAVID B. PARFITT, ${ }^{b}$ \\ AND SARA KOLLACK-WALKER ${ }^{c}$ \\ ${ }^{b}$ Department of Anatomy and Cell Biology \\ University of Michigan \\ Ann Arbor, Michigan 48109-0616 \\ ${ }^{c}$ Mental Health Research Institute \\ University of Michigan \\ Ann Arbor, Michigan 48109-0720
}

\begin{abstract}
Experimental efforts to define the functional neuroanatomical circuitry underlying male sexual behavior in rodents have relied in the past on the observation of behavioral deficits in males with selectively placed brain lesions. More recently, experiments in our laboratory and several other laboratories have attempted to selectively stimulate or eliminate Fos protein production associated with mating by controlling either the past sexual experience of the animal or the stimuli and behavioral events immediately preceding perfusion-fixation of the brain, ${ }^{1-5}$ by altering the hormonal status of the behaving animal, ${ }^{6}$ or by observing Fos-ir patterns ipsilateral to unilateral lesions in key nuclei of the mating behavior pathway. ${ }^{4}$ In this chapter we summarize the results of several studies in the male Syrian hamster in light of previously reported behavioral effects of lesions in the same areas.
\end{abstract}

\section{MATING BEHAVIOR CIRCUITS REVEALED BY LESION STUDIES}

In 1970 Murphy and Schneider ${ }^{7}$ reported that olfactory bulbectomy, which destroys both olfactory and vomeronasal inputs to the CNS, completely eliminated male sexual behavior and greatly diminished intermale aggression in golden hamsters and that these effects were not secondary to reduced testosterone stimulation of the brain. Subsequent studies indicated that the peripheral sensory afferents and the centrally projecting efferents of both the main (olfactory) and accessory (vomeronasal) bulbs

\footnotetext{
${ }^{a}$ This research was supported by the National Institutes of Health (NINCD-NS 20629 and the Morphology Core Facility of NICHD-P-30, HD 18258).

${ }^{d}$ Present address: Department of Psychology, Uris Hall, Cornell University, Ithaca, New York 14853.
} 


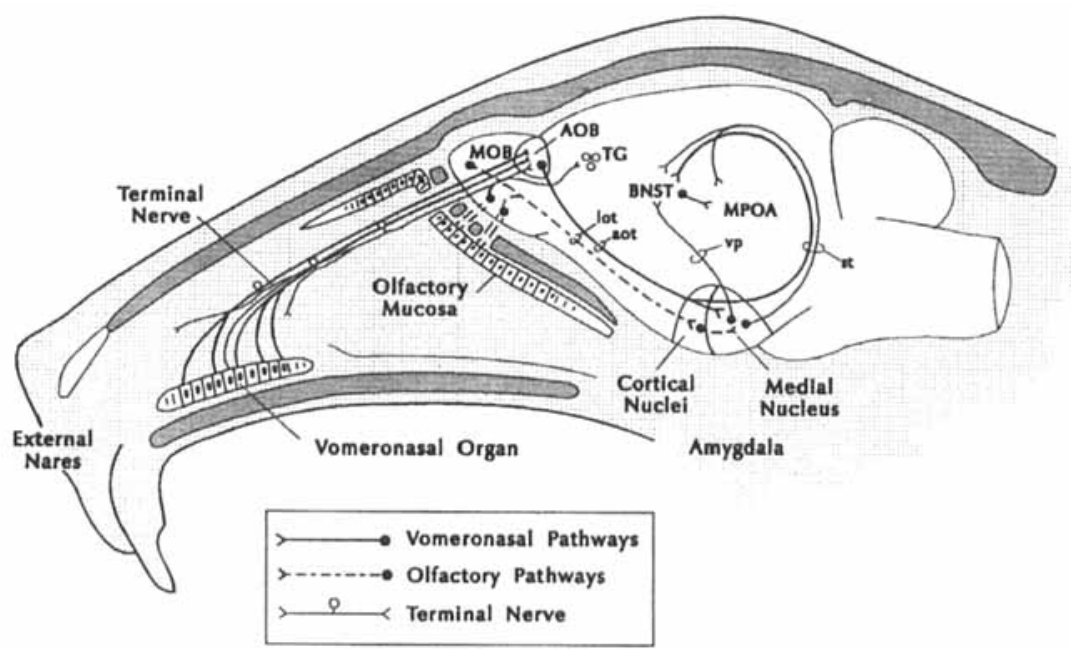

FIGURE 1. Sagittal view of a hamster head illustrating the chemosensory pathways critical to male hamster sexual behavior. Abbreviations: $A O B=$ accessory olfactory bulb; aot = accessory olfactory tract; $\mathrm{BNST}=$ bed nucleus of the stria terminalis; lot $=$ lateral olfactory tract; $\mathrm{MPOA}=$ medial preoptic area; $\mathrm{st}=$ stria terminalis; $\mathrm{TG}=$ terminal ganglion; $\mathrm{vp}=$ ventral pathway.

were contributing to the maintenance of mating behavior ${ }^{8-11}$ and that the essential chemosensory pathways included the lateral olfactory tract projections to the corticomedial amygdala. ${ }^{12}$

Further analysis of behavior after amygdaloid lesions focused attention on the anterior part of the medial nucleus of the amygdala (MeA), where damage mimicked the effect of olfactory bulbectomy and completely abolished both phases of sexual behavior, the chemoinvestigatory phase (sniffing and licking the female's head, flanks and, anogenital area) and the copulatory phase (mounts, intromissions and ejaculations). In contrast, males with lesions of the posterior part of this nucleus, $\mathrm{MeP}$, exhibited partial reduction in chemoinvestigation, with no deficits in copulatory behavior; however, they did show changes in the temporal pattern of copulation. ${ }^{13}$ Neither lesions in the basolateral amygdala nor those in other nuclei of the corticomedial amygdala produced these effects. ${ }^{14,15}$

From the medial nucleus the behaviorally relevant efferents follow both the stria terminalis and the ventral amygdaloid pathway ${ }^{16-18}$ to the bed nucleus of the stria terminalis BNST and the medial preoptic area (MPOA). These pathways are illustrated in FIGURE 1. However, unlike the effects of damage to the medial amygdaloid nucleus, lesions in the BNST and MPOA revealed a dissociation of effects on the two major components of the male hamster's mating activities. Whereas some animals with lesions of either the BNST or the MPOA showed severe deficits in both phases of mating, many animals with BNST damage showed a deficit in chemoinvestigation without any alteration in copulation, and the reverse effect was observed after lesions of the MPOA.$^{19}$ The differential effects of these lesions in the chemosensory pathways are summarized in FIGURE 2. 

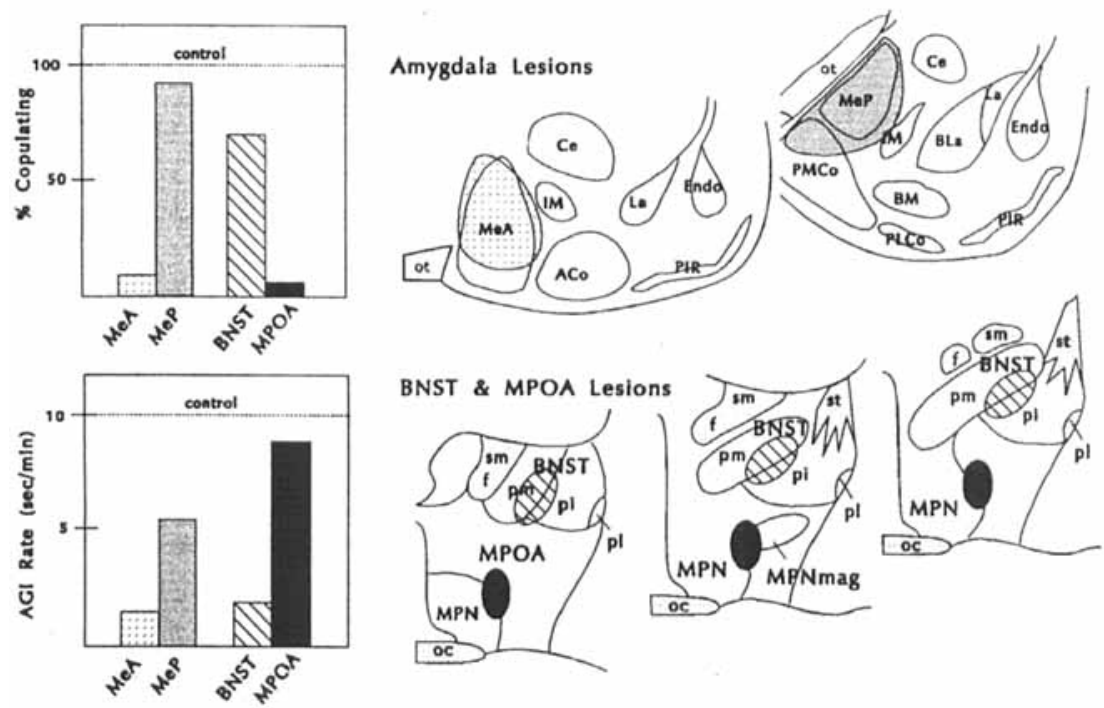

FIGURE 2. Effects of lesions placed within the anterior or posterior subdivisions of the medial amygdaloid nucleus (MeA or MeP), bed nucleus of the stria terminalis (BNST), or medial preoptic area (MPOA) on the percentage of males copulating with receptive females and on the rate of investigation of the female's anogenital region (AGI). Each shaded or patterned area represents the area that was damaged in common in a group of males. See TABLE 1 for additional abbreviations.

\section{MATING-INDUCED EXPRESSION OF FOS PROTEIN}

\section{Fos-Immunoreactivity Reveals Activation of Neurons Not Only in Areas Where Lesions Eliminate the Behavior, But Also in Areas Where Lesions Have Subtle Effects}

The observations of Robertson et al., ${ }^{5}$ that immunohistochemistry for Fos protein could be used to visualize neuronal activation after male sexual behavior in the rat, presented an opportunity to localize and study directly the neurons activated as a result of mating in the Syrian hamster. In the first experiment in this species, in which each sexually naive male mated for 55 minutes with three females, ${ }^{1}$ Fos immunoreactivity (Fos-ir) was elevated above control levels in numerous nuclei within the corticomedial amygdala, BNST, and MPOA (FIGs. 3 and 4). Furthermore, quantitative analysis revealed a broad range of differences across nuclei, from $35 \%$ more Fos-ir cells in the posterolateral cortical nucleus of the amygdala to a 20 -fold difference, or a $2000 \%$ increase, in the posteromedial BNST.

It was immediately clear, however, that no correlation existed between the magnitude of the increase in Fos-ir cells in a given area and the significance of that area, as determined by lesions, for initiation of sexual behavior. In the $\mathrm{MeA}$, where bilateral lesions completely eliminate both phases of the behavior, ${ }^{15}$ the number of Fos-ir cells 
A

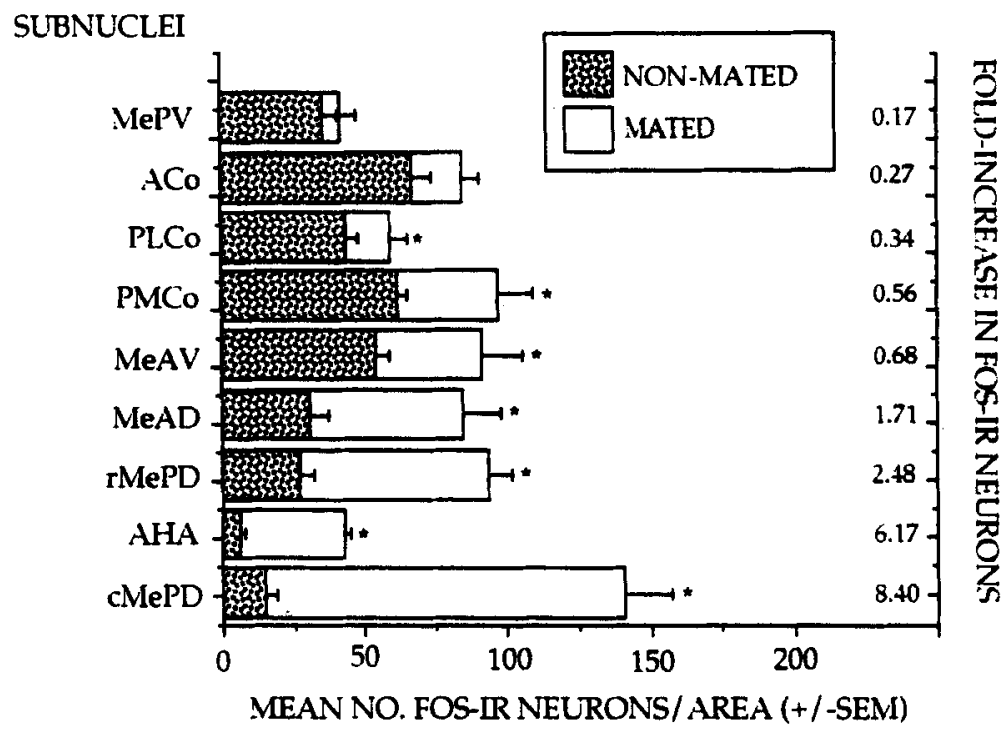

B

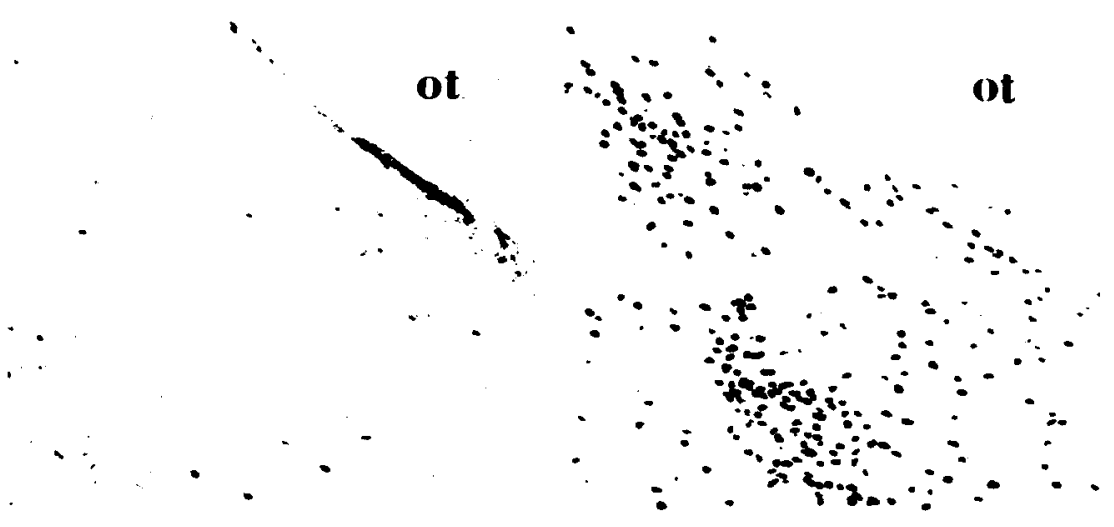

FIGURE 3. (A) Mean number of Fos-ir neurons per $0.21 \mathrm{~mm}^{2}$ in subnuclei of the amygdala in nonmated $(n=5)$ and mated $(n=6)$ groups of male hamsters. The fold-increase in Fos-ir neurons indicates the mating-induced increase above control levels. $*=$ area that contained significantly more Fos-ir neurons following mating. For abbreviations see TABLE 1. (B) Photomicrographs of Fos-ir neurons in the caudal posterodorsal division of the medial nucleus of the amygdala (cMePD) of nonmated (left) and mated (right) hamsters. ot $=$ optic tract. 
A

\section{SUBNUCLEI}

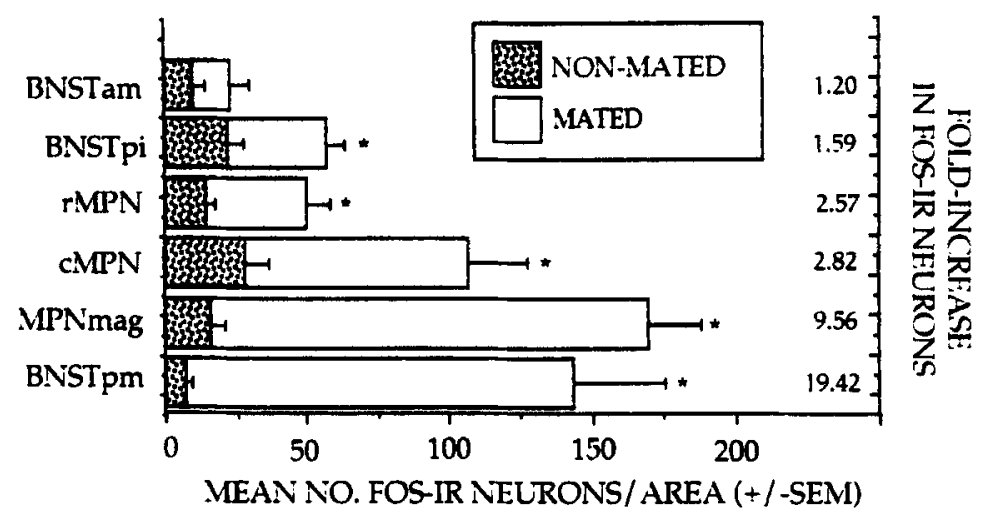

B
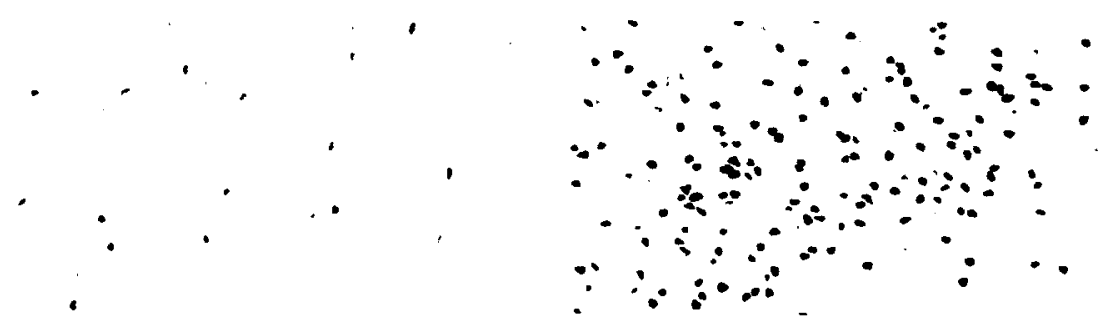

FIGURE 4. (A) Mean number of Fos-ir neurons per $0.21 \mathrm{~mm}^{2}$ in subnuclei of the bed nucleus of the stria terminalis and medial preoptic area in nonmated $(n=5)$ and mated $(n=6)$ groups of male hamsters. The fold-increase in Fos-ir neurons indicates the mating-induced increase above control levels. $*$ = area that contained significantly more Fos-ir neurons following mating. For abbreviations see TABLE 1. (B) Photomicrographs of Fos-ir neurons in the magnocellular medial preoptic nucleus (MPNmag) of nonmated (left) and mated (right) hamsters.

was elevated only $100 \%$ above control levels in the mated males (FIG. 3), while in the same animals an $800 \%$ increase was observed in the MeP, where lesions alter only the temporal pattern of copulation. ${ }^{13}$ Similarly, in the caudal MPOA, where lesions abolish the copulatory phase of the behavior, ${ }^{19}$ we observed a $1000 \%$ difference (FIG. 4), whereas in the posteromedial BNST (BNSTpm), where lesions interfere with the chemoinvestigatory phase of the behavior, ${ }^{19}$ mated males had almost $2000 \%$ more Fos-ir cells than did controls. Finally, a $200 \%$ increase in Fos-ir was observed in a specific area of the paraventricular nucleus of the hypothalamus, a nucleus with no known involvement in sexual behavior. Although these results indicated that Fos-ir could be detected in brain regions shown previously to be important in male sexual 
behavior, it was unclear whether this pattern reflected activity associated selectively with male copulation or associated with social behaviors in general.

\section{Comparison of Fos-ir after Mating and Agonistic Behavior Reveals Activation in a "Mating-Specific" Circuit and in a "Nonspecific" Circuit Activated Equally by Mating and Aggression}

\section{Identification of the "Mating-Specific" Circuit}

To narrow the investigation to neurons specifically activated by mating in the male, we compared the Fos-ir pattern in three groups of sexually naive males that had lived in isolation for 1 week and on the day of the experiment either (1) engaged in sexual behavior with a receptive female or (2) agonistic behavior with a male or (3) were left alone after handling by the experimenter (Experiment 2). All of these males remained in their own cages during behavioral testing. ${ }^{2}$ The rationale for this experiment was based on the observation that both mating and aggression are diminished by removal of the olfactory bulbs ${ }^{7.20}$ and by bilateral lesions of the amygdala $^{21}$ and on the assumption that both behaviors require emotional arousal, autonomic activation, and strenuous motor activity on the part of the males, whereas the specific chemosensory stimuli and motor patterns are different. We therefore hypothesized that we could identify mating-specific areas of the limbic system by subtraction of the areas activated equally by the two behaviors, recognizing that in doing so we might be eliminating from further consideration areas in which equal but different populations of cells in one region are active during the two different social behaviors.

Analysis of 26 limbic areas in these male hamsters showed significant increases in Fos-ir above control levels in 20, but in only 4 of these 20 areas was Fos-ir increased in the copulating males and not at all in the agonistic males. All four of these nuclei were in the preoptic region, including medial preoptic nucleus (MPN), the magnocellular medial preoptic nucleus (MPNmag, a small nucleus in the lateral part of the medial preoptic area of the hamster ${ }^{22}$ ), the dorsolateral medial preoptic area (MPOA), and the preoptic portion of the BNST (the posteroventral portion of the posteromedial BNST or BNSTpm[pv]). These results are summarized in FiGURE 5.

In addition to these selectively activated nuclei in the preoptic region, two nuclei were activated by both mating and agonistic behavior, but to a greater degree by mating. Both the lateral part of the caudal posterodorsal division of the medial amygdaloid nucleus (cMePD) and the rostral part of the posteromedial BNST (BNSTpm[ad]) contained 2-3 times as many Fos-ir cells in the copulating males as in the fighting males, although the Fos-ir cell populations were intermingled and indistinguishable by location in both areas. However, in addition, an easily distinguishable group of cells, close to the optic tract in cMePD, was always labeled in the brains of mated but not aggressing males (Frgs. 5 and 6). The behavioral significance of this activation pattern in BNSTpm(ad) and cMePD was revealed by work in other laboratories and confirmed in our recent studies, and we will return to this topic.

Of the six areas that were significantly activated by mating (MPN, MPNmag, MPOA, BNSTpm[ad], BNSTpm[pv], and cMePD), four belong to a neuroanatomical 


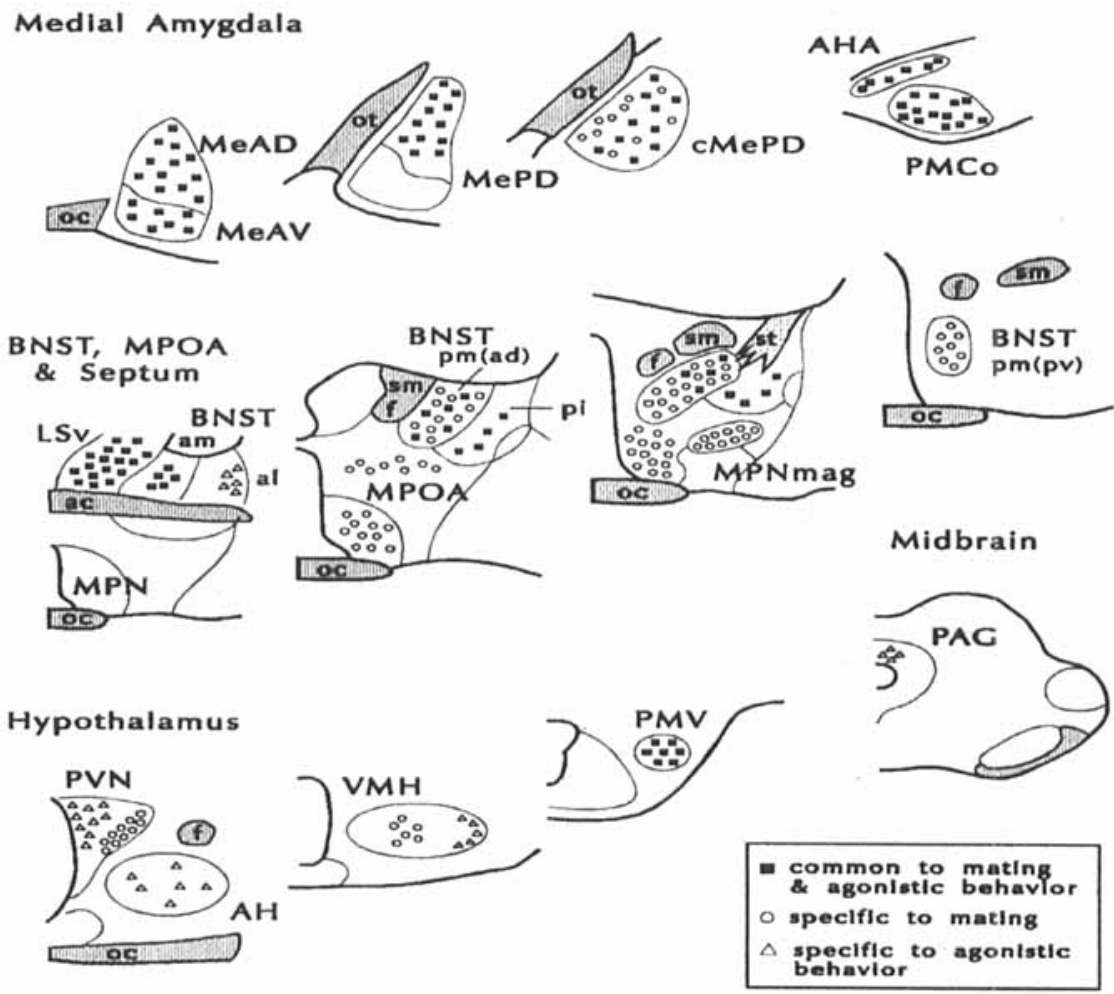

FIGURE 5. Summary of the net effect of copulation and agonistic behavior on the pattern of Fos-immunostaining within limbic regions of the male Syrian hamster brain. The intermingling of "common" and "mating-specific" activation symbols in BNSTpm and in cMePD reflects activation of neurons above control after both behaviors, but with mating producing significantly greater numbers of Fos-ir neurons than agonistic encounters. For abbreviations see TABLE 1.
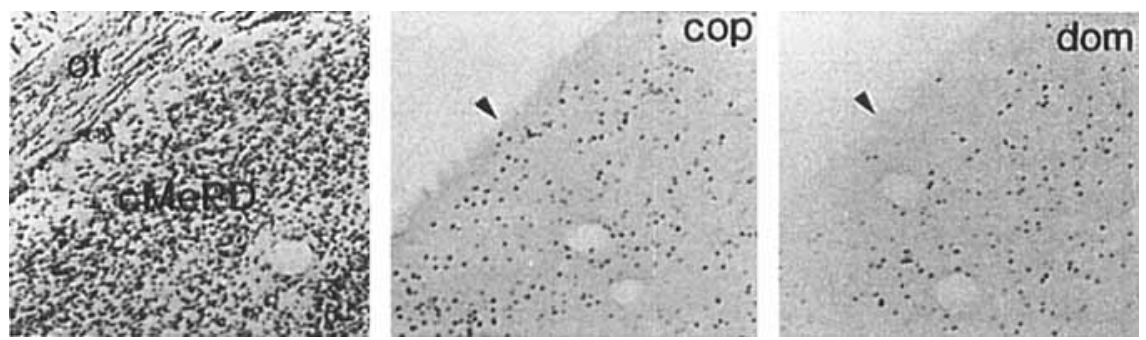

FIGURE 6. Photomicrographs of the caudal posterodorsal subdivision of the medial amygdaloid nucleus (cMePD), illustrating the cytoarchitecture of this subnucleus in a cresylvioletstained section and the distribution of Fos-ir neurons within this area in a representative animal from copulator (cop) and dominant (dom) groups. Arrowhead identifies the boundary between the molecular layer of $\mathrm{cMePD}$ and the optic tract (ot). 
circuit that can be defined in the hamster ${ }^{23}$ and rat $^{24}$ by the projections of the posterior division of the medial amygdaloid nucleus (MePD). Iontophoresis of PHA-L into MePD of the hamster reveals projections to the BNSTpm (including BNSTpm[ad] and BNSTpm[pv]) and the medial region of the MPOA, including the MPN. Furthermore, these are the subdivisions of Me, BNST, and MPOA that are most densely populated with androgen- and estrogen-receptor producing neurons, ${ }^{25-29}$ where delivery of gonadal steroids can reinstate sexual behavior in castrated males that have stopped mating. ${ }^{30}$

\section{Specific Antecedents for Fos Production within the "Mating-Specific" Circuit}

With evidence that structures in a neuroanatomically definable circuit of sex steroid-sensitive areas were selectively activated by sexual behavior, we were anxious to try to determine the specific components of mating that induced Fos production in each of these areas. In our next experiment (Experiment 3), six groups of six male hamsters were given prior mating experience in a testing arena. On the day of the experiment all males were placed in the mating arena. Males in the handled-control group with experience (HC-Exp) were left alone. A second group received female hamster vaginal secretion (FHVS) presented on a cotton swab. The remaining groups were given free access to a receptive female, but their mating behavior was interrupted after either five intromissions (5-INTRO), one ejaculation (1-EJAC), five ejaculations (5-EJAC), or the first long intromission (Long-INTRO). (In the male hamster, the long intromission at the termination of mating is an extended period of intromissions with repeated pelvic thrusting, but without ejaculation. ${ }^{31,32}$ ) A seventh group of six males, that had no prior mating experience but had been placed in the clean testing arena alone on pretest days (HC-Naive), were again placed in the arena on the day of the experiment.

The results from this third experiment provided both new data and data confirming the results from other laboratories. ${ }^{3,433,39,40}$ Quantification of the number of Fos-ir neurons per nuclear area was analyzed using a one-way ANOVA and post hoc comparisons with the Tukey test for significant differences. This analysis identified discrete patterns of activation in association with specific aspects of male mating behavior, including sexual conditioning, pheromonal stimulation, chemosensory investigation of the female, copulation, and sexual satiety.

Sexual Conditioning. Significant differences in Fos-ir cell numbers were found in cMePD, MPN, and MPNmag of sexually experienced and naive control males (FIGs. 7 and 8, Exp 3). On the day of the experiment these males were treated identically; both were placed in the empty testing arena for an hour. The only difference was that the experienced controls had mated on three previous occasions in an identical arena, whereas the naive males had simply been placed in the empty arena on those occasions. This sexual experience-associated neuronal activation presumably reflects a conditioned response to environmental cues and may be the basis of previously documented changes in mating behavior following experience. ${ }^{34,35}$ Alternatively or in addition, it may indicate brain areas that regulate neuroendocrine reflexes that are known to be enhanced after sexual encounters. ${ }^{36-38}$

Pheromonal Stimulation and Chemosensory Investigation of the Female. The second distinctive activation pattern within this circuit is associated with pheromonal 

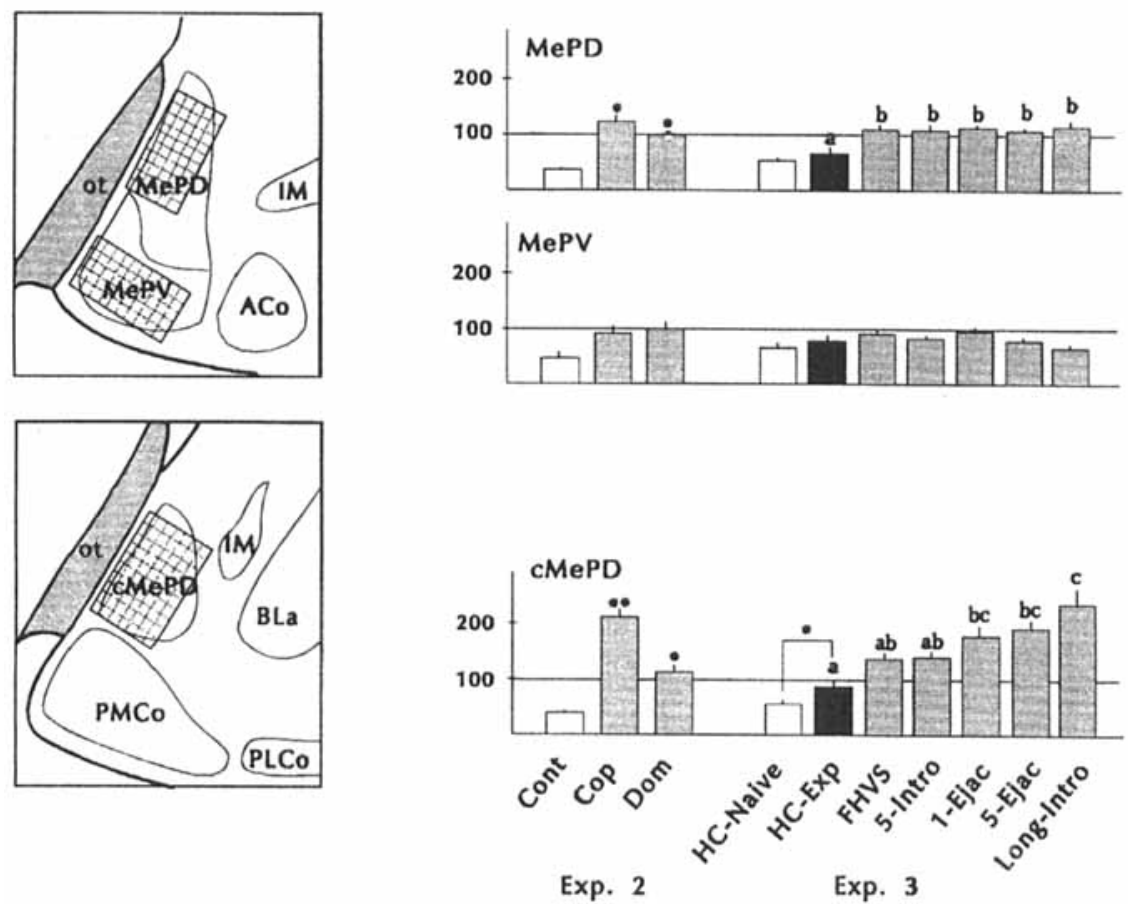

FIGURE 7. Location of cell counts within the posterior subdivisions of the medial nucleus of the amygdala and the resultant mean number of Fos-ir neurons ( \pm SEM) per subdivision for control (Cont), copulator (Cop), and dominant (Dom) groups in Experiment 2 and for Handled Control-Naive (HC-Naive), Handled Control-Experienced (HC-Exp), female hamster vaginal secretion (FHVS), 5-Intromissions (5-Intro), 1-Ejaculation (1-Ejac), 5-Ejaculations (5Ejac), and Long-Intromission (Long-Intro) groups of males in Experiment 3. A significant increase in the number of Fos-ir neurons (above Cont in Exp 2; HC-Exp above HC-Naive in Exp 3) is indicated with an asterisk $(p<0.05)$. In Experiment 3 a significant difference $(p$ $<0.05$ ) between groups (excluding HC-Naive) is identified by different lowercase letters.

stimulation. In 1993 Fiber et al. ${ }^{39}$ reported that female hamster vaginal secretion (FHVS) induced Fos production in the areas we designated MePD, BNSTpm(ad), and MPNmag but not in the MPN. Fernandez-Fewell and Meredith ${ }^{3}$ also observed activation after FHVS alone in the MePD and BNSTpm(ad) (their "mBNSTpc"). They did not report on MPNmag, but, like Fiber et al. ${ }^{39}$ they found that MPN was not activated. Our study replicated these findings in MePD (FIG. 7), MPNmag (FIG. 8), and BNSTpm(ad) (FIG. 9). In addition, our histological material presented a distinctive qualitative difference in the location of Fos-ir neurons in cMePD between control animals and all other groups, including both FHVS-stimulated and mated males. Illustrations in Fiber et al. ${ }^{39}$ and Fernandez-Fewell and Meredith ${ }^{3}$ also show this distinctive pattern of immunolabeling. In these two reports and in all three of our studies, a strip of cells close to the optic tract in $\mathrm{CMePD}$ of the medial amygdaloid nucleus is activated in all animals that have been exposed to FHVS (FIG. 6). 

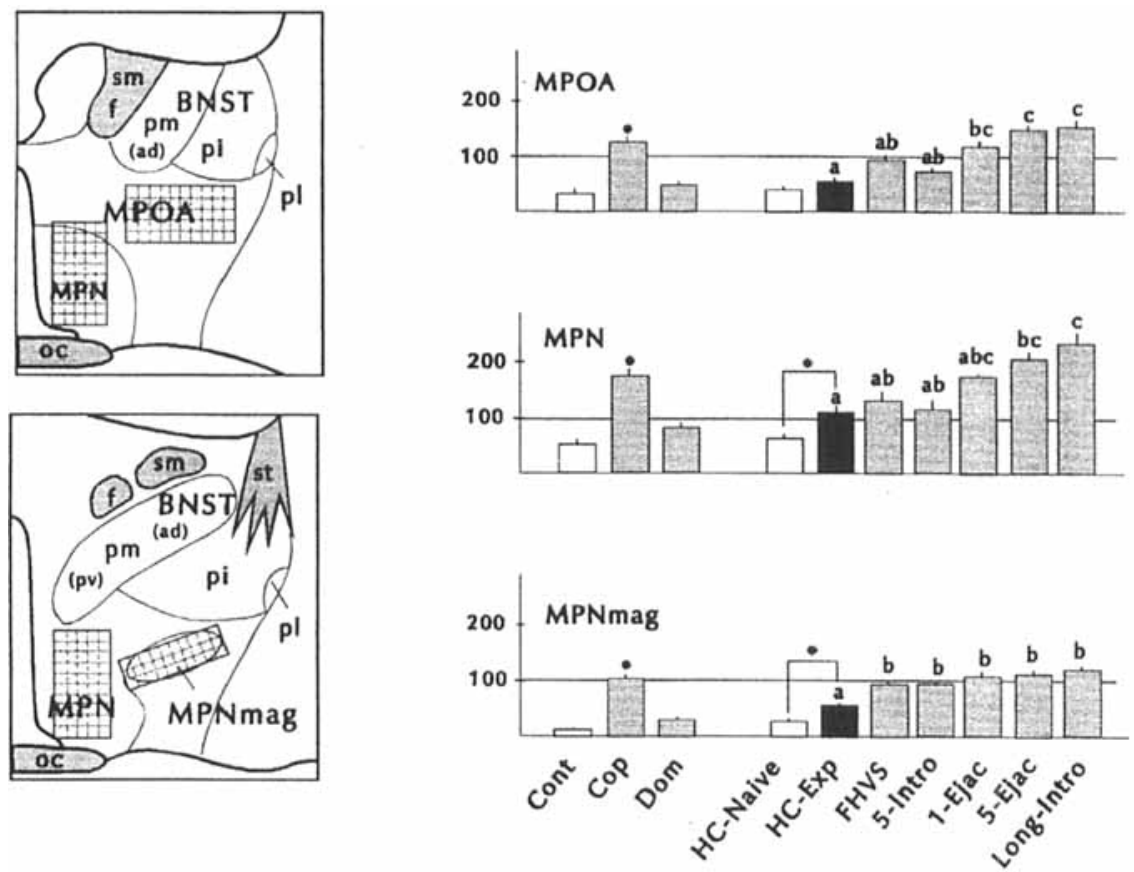

Exp. 2

Exp. 3

FIGURE 8. Location cell counts and mean number of Fos-ir neurons counted within the medial preoptic area. For explanation see legend for Figure 7.

Although each of these three areas is activated by the presentation of FHVS alone, the functional significance of this pheromonal stimulation may be different. Whereas lesions of MePD reduce anogenital investigation of the female (AGI) and affect only the temporal pattern of copulation, small lesions of BNSTpm(ad) can virtually eliminate AGI without altering copulation, and lesions that include the MPNmag can abolish copulatory behavior without affecting AGI. Thus, it would appear that projections from MePD to BNSTpm may process FHVS-associated information to drive the chemoinvestigatory behavior. Additional evidence to support this hypothesis comes from Fernandez-Fewell and Meredith ${ }^{3}$ who report that male hamsters in which the vomeronasal organ has been removed prepubertally (at 17 days of age) show no Fos-ir induction in BNSTpm(ad) (their mBNSTpc) when exposed to FHVS, but do show statistically significant increases in Fos-ir if they engage in chemoinvestigatory behavior with a receptive female, regardless of whether they show any copulatory responses.

In the MPNmag, in contrast, FHVS stimulation may be required for the pheromonal elicitation of mounts, intromissions, and ejaculations. Although this empirically derived explanation is clear and consistent with the hamster's dependence on chemosensory inputs for mating, the specific pathways that might be responsible for mediating these pheromonal effects in MPNmag have not been identified. 

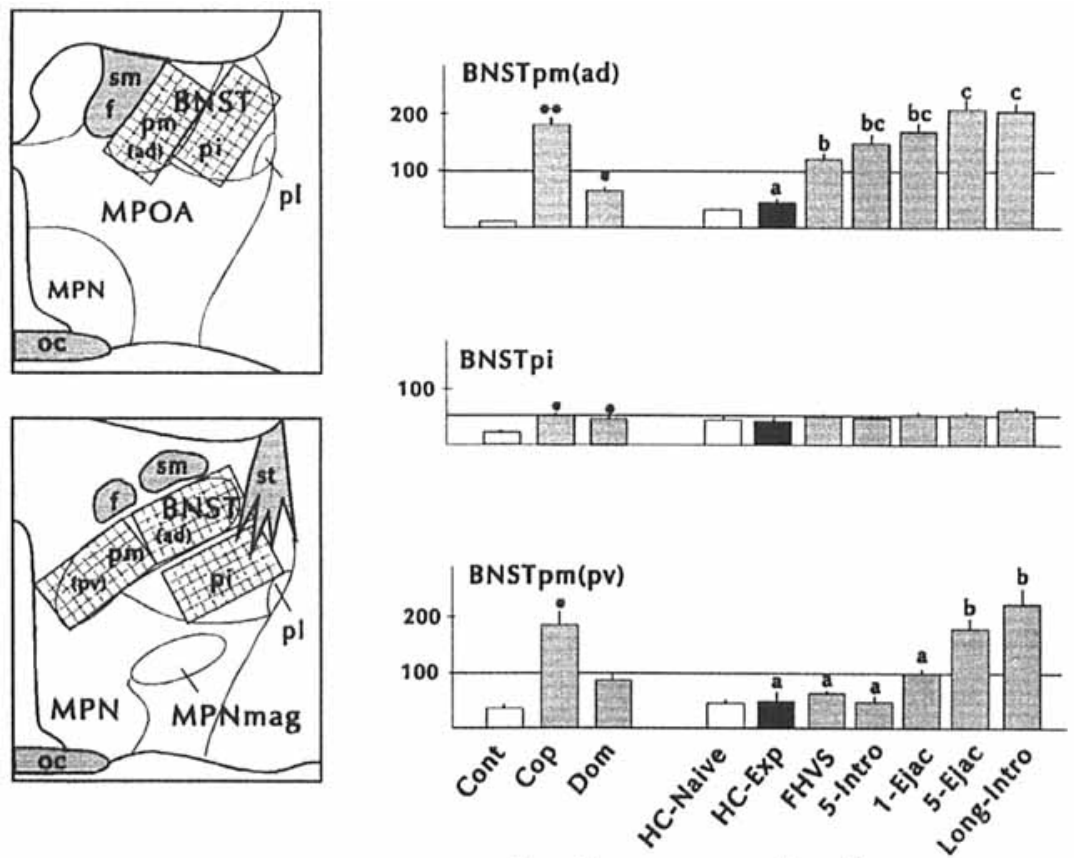

Exp. 2

Exp. 3

FIGURE 9. Location cell counts and mean number of Fos-ir neurons counted within the bed nucleus of the stria terminalis. For explanation see legend for FIGURE 7.

Copulation. Mating to intromissions did not selectively increase Fos production in any of the areas we analyzed. However, in males that were allowed to mate to ejaculation, several areas along this pathway, including cMePD, BNSTpm(ad), BNSTpm(pv), MPOA, and MPN, and the central tegmental field of the midbrain (CTF), appeared to reach a new level of activation. In our sexually experienced animals this represented a further significant increase in the number of Fos-ir cells, adding to the populations in $\mathrm{cMePD}$ and MPN that had already been stimulated to express Fos on exposure to the testing arena. However, in both of these areas, and in BNSTpm(ad), what was actually observed was a gradual and continual increase in the levels of activation, rather than an abrupt increase associated with ejaculations (FIGS. 7, 8, and 9). Only in BNSTpm(pv) (FIG. 9) and the CTF did it appear that the population was stimulated for the first time in the behavioral sequence by the occurrence of ejaculations. This is in agreement with the observations of Coolen ${ }^{40}$ in the male rat, but in contrast to the findings of Baum and Everitt, ${ }^{4}$ who reported the induction of Fos protein in the CTF after five intromissions.

Sexual Satiety. Finally, investigations of Fos-ir in the male hamster after copulation have produced evidence for selective activation of cell "clusters," small groups of cells (approximately 150-250 cells in an area no more than $250 \mu \mathrm{m}$ in diameter), that have not, to date, been observed as discrete populations with any other markers 
or histological methods (FIG. 3). These cells were reported in cMePD by Kollack and Newman ${ }^{1}$ and by Fernandez-Fewell and Meredith $^{3}$ in cMePD and rostral BNST (corresponding to rostral BNSTpm[ad] in this paper; called mBNSTpr by FernandezFewell and Meredith). In both of these studies each male mated for at least 45 minutes with three females. In Experiments 2 and 3 described above, ${ }^{2,42}$ we determined that these Fos-ir clusters of cells are readily distinguishable in $40 \mu \mathrm{m}$ sections of cMePD and BNSTpm(ad) only in males that had mated to multiple ejaculations. They were never present in males that had been stopped after one ejaculation but were found in one half to two thirds of males that had achieved five ejaculations, and in all males that had mated to long intromissions.

These findings led one of us (D.B.P.) to hypothesize that the appearance of the Fos-ir cell clusters was associated with the culmination of a mating behavior sequence and not with multiple ejaculations per se. In the first of two experiments he compared the brains of males that were mated for 4 consecutive days to long intromissions or rested and then mated only on the fourth day to long intromissions. The rationale for this paradigm was based on observations by Huck and Lisk, ${ }^{41}$ who showed that rested male hamsters (i.e., animals that have not mated for at least 2 weeks) will ejaculate an average of 13 times before reaching long intromissions, whereas males mated on succeeding days will reliably ejaculate only four times, on average, before stopping. Thus, this study was designed to determine if the number of ejaculations preceding long intromissions influences the appearance of clusters in cMePD.

In six rested male hamsters mated to long intromissions, after an average of 10 ejaculations, all 6 brains had clusters in cMePD. A second group of males were mated to long intromissions for 4 consecutive days and averaged only four ejaculations on the fourth day, but five of six males in this group also had Fos-ir clusters in the cMePD. In a third group, male hamsters mated to long intromissions for 3 consecutive days were placed in an empty clean cage on the fourth day to serve as controls, and none of these males showed the cell clusters. These results were consistent with the hypothesis that activation of cMePD cell clusters is correlated with the onset of sexual satiety rather than the number of ejaculations per se. However, because all animals that exhibited clusters had ejaculated more than once, we could not exclude the possibility that cluster activation was dependent on multiple ejaculations.

As already noted, we had never seen this activation pattern in rested male hamsters stopped after one ejaculation. Therefore, in a second experiment one of us (DBP) compared the Fos-ir pattern in a group of rested animals that had been interrupted after one ejaculation with the pattern in a second group of males that were mated to long intromissions for 3 consecutive days and then interrupted after one ejaculation on the fourth day. Although none of the rested animals had Fos-ir cell clusters, five of six males in the second group did (FIG. 10). Therefore, we conclude that the appearance of cell clusters in cMePD does not depend on multiple ejaculations, but is associated with the onset of sexual satiety.

In contrast to the neuronal activation observed along the optic tract in the medial part of cMePD, which appears following exposure to FHVS (FIG. 6), activation of the cell clusters in the lateral part of this same region may be independent of chemosensory stimulation. Fernandez-Fewell and Meredith ${ }^{3}$ report that these lateral Fos-ir cell clusters were present in the brain of one male that mated following removal of the vomeronasal organ ( 7 of 9 animals in this group did not mate). Instead, based 


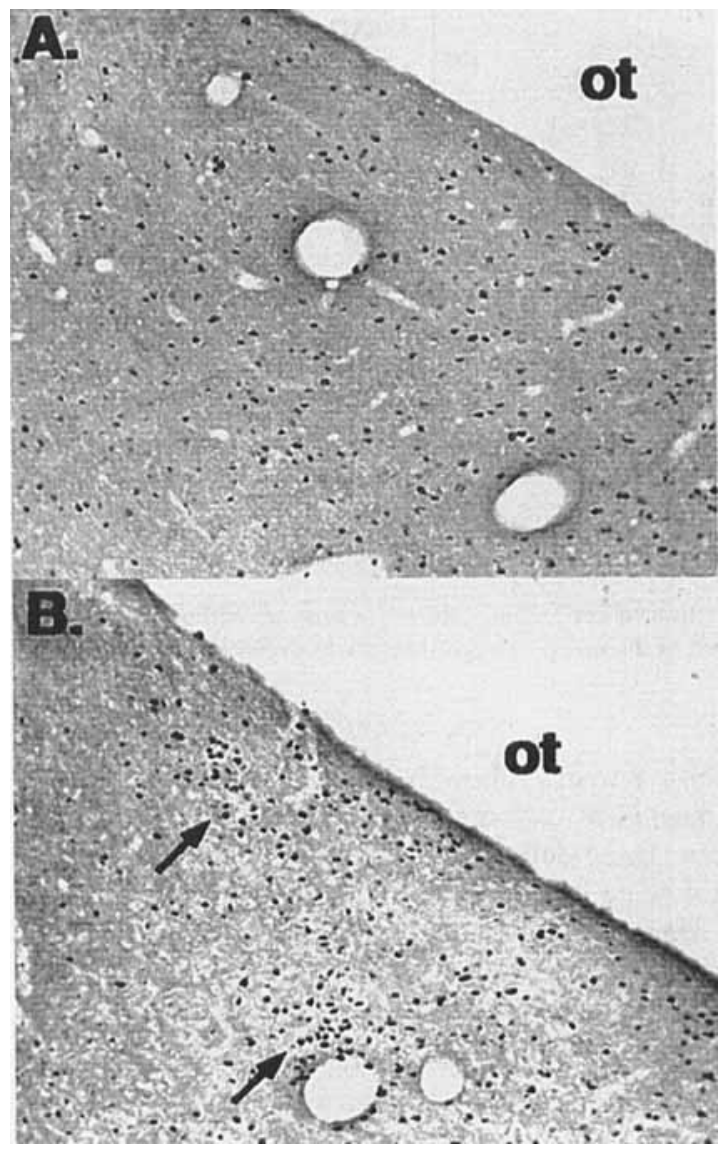

FIGURE 10. Photomicrographs of Fos-ir neurons within the cMePD of a rested male hamster stopped after one ejaculation (A) and a male hamster mated to one ejaculation on the fourth of 4 consecutive days of mating to long intromissions (B). Arrows indicate the location of Fos-ir cell clusters.

largely on data from the male rat, it appears more likely that these cell clusters are activated by somatosensory stimuli and/or visceral stimuli processed through ascending spinal cord pathways reaching the amygdala via the midbrain, where a field of cells in the ventral tegmentum show Fos-ir after copulation in both the rat ${ }^{4,40}$ and the hamster. ${ }^{42}$

Given the observations that within the cMePD there may be separate cells groups activated specifically by FHVS, sexual conditioning, copulation to ejaculation, and sexual satiety, it is surprising that lesions destroying this area do not eliminate any of the behavioral components of male sexual behavior. These lesions cause decreases in chemoinvestigatory behavior and an increase in latency to the first ejaculation in 

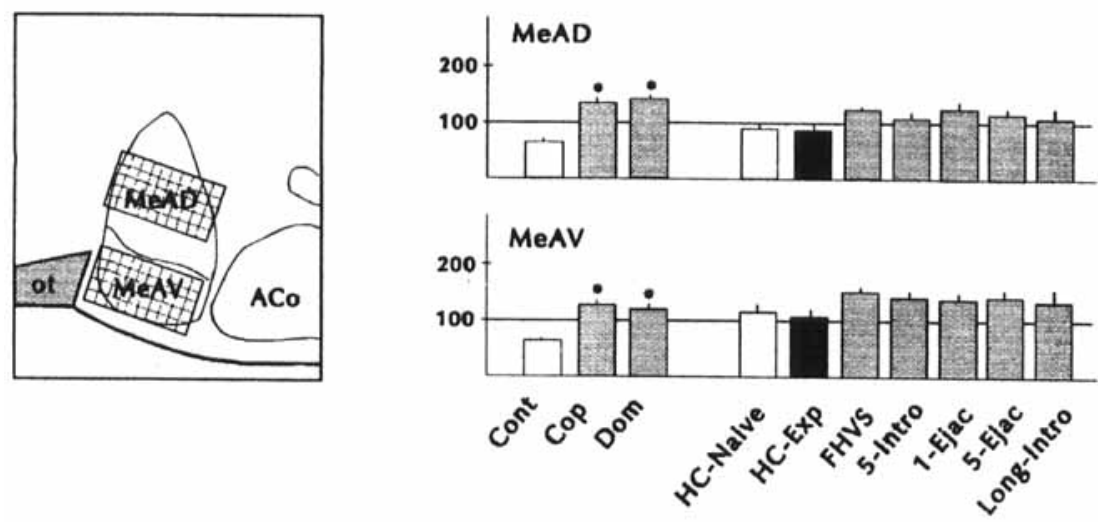

Exp. 2

Exp. 3

FIGURE 11. Location of cell counts and mean number of Fos-ir neurons counted within the anterior subdivisions of the medial amygdaloid nucleus. For explanation see legend for FIGURE 7.

a series, ${ }^{13}$ but over 4-8 weeks following the lesions the only behavioral changes that have been documented to date are changes in the temporal pattern of mating by experienced males. These deficits appear insignificant when compared to the total loss of mating following lesions of the MeA. However, work by Lanier et al. ${ }^{43}$ and Huck and Lisk ${ }^{44}$ suggests that the timing of the copulatory bout, which in the Syrian hamster is controlled largely by the male, and particularly the occurrence of long intromissions, are of great importance for successful conception, the ultimate goal of mating.

\section{A Separate "Nonspecific" Circuit May be an Essential "Arousal Circuit" for Social Behaviors}

In contrast to the projections of MePD, axons of the cells in MeA preferentially terminate in the posterointermediate BNST (BNSTpi) and the lateral part of the MPOA including MPNmag. ${ }^{23}$ It was the integrity of this circuit which the results of lesion studies had indicated was absolutely essential for mating to occur in the hamster. ${ }^{15.19}$ What did the results of Fos expression after mating or agonistic behavior tell us about this circuit? Quantitative analysis of the data indicated that cells in MeA and BNSTpi were equally activated after these two behaviors (FIGs. 9 and 11, Exp 2 ), and histological analysis indicated that the activated cells were equally distributed. Acknowledging that future research might reveal separate but equal, intermingled populations of activated cells in these two circumstances, we tentatively concluded that the Fos-ir cells in MeA and BNSTpi belong to an arousal circuit, providing essential background activation for discrete stimuli to elicit appropriate social responses.

This conclusion would lead to the prediction that any animal engaging in mating would show increased Fos-ir in these areas, but quantitative analysis of Fos-ir neurons 
in MeA (MeAD and MeAV) and BNSTpi in the seven groups of males in Experiment 3 revealed no significant increase in any of the stimulated groups of animals relative to controls (FIGS. 9 and 11, Exp 3), even though comparison of the data from Experiments 2 and 3 revealed that the number of Fos-ir cells in the mated males were equivalent. This comparison indicated that an increase in the number and variance of immunolabeled cells in the control groups (HC-Exp and HC-Naive) in Experiment 3 was, in fact, responsible for the failure to replicate the results of Experiment 2. FIGURE 12 presents data from individual animals in these two experiments, showing comparable levels of activation in the copulators from Experiment 2 and the males mated to five ejaculations in Experiment 3, but a striking difference between the number of Fos-ir neurons in the control groups in these two experiments.

Although the basis for the apparent changes in Fos production in the control animals remains to be investigated, the explanation may be attributable to differences in the amount of prior handling and in the location where the experiments were conducted. In the first study in this series and in Experiment 2, males were handled by the experimenter only briefly ${ }^{2}$ or not at all ${ }^{1}$ before being anesthetized for perfusion fixation of the brain. In these experiments the control, mated, and dominant animals all remained in their home cages. In the third study all males were handled on three testing days before the day of the experiment and again on the day of the experiment, when in each case all males were placed in a testing arena. Although it might be argued that repeated handling and experience in the arena should have acclimated the animals to this procedure, the fact remains that all of these animals spent the hour before anesthetization in a testing arena, whereas all of the animals in the first two experiments were in their home cages during this period. We hypothesize that being placed in the arena aroused both control and stimulated animals in the third experiment sufficiently to eliminate differences between these groups in a circuit that is active during arousal.

\section{CONCLUSIONS}

Exposure of male Syrian hamsters to female pheromones, or copulation with the female, increases immunohistochemically detectable Fos protein and Fos-related antigens in a number of discrete populations of limbic system neurons where lesions eliminate male sexual behavior. Although in the initial stages of this work it was tempting to conclude that Fos-immunoreactive cell populations in the lesion-sensitive areas are essential for the initiation of mating, it is now evident that Fos immunoreactivity is also elevated in regions where lesions have less impact on the occurrence of the behavior. Furthermore, no correlation appears to exist between the magnitude of the increase of Fos-ir in a given area and the significance of that area, as determined by lesions, for initiation of mating. Perhaps areas essential for triggering the behavior are activated by appropriate stimuli only briefly, at the onset of the behavior and, depending on the previous experience of the individual animal, are responsible for activating other circuits that pace and maintain the events of copulation. We must also keep in mind, however, that the Fos-defined neuroanatomical substrate does not necessarily represent the entire circuitry underlying mating, because many, but by no means all, neurons employ $c$-fos as a transcription factor following excitation. 


\section{MeAD}
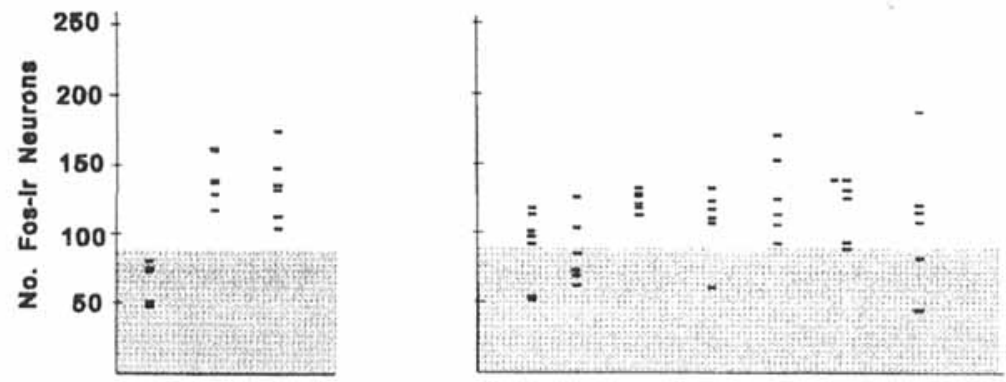

\section{MeAV}
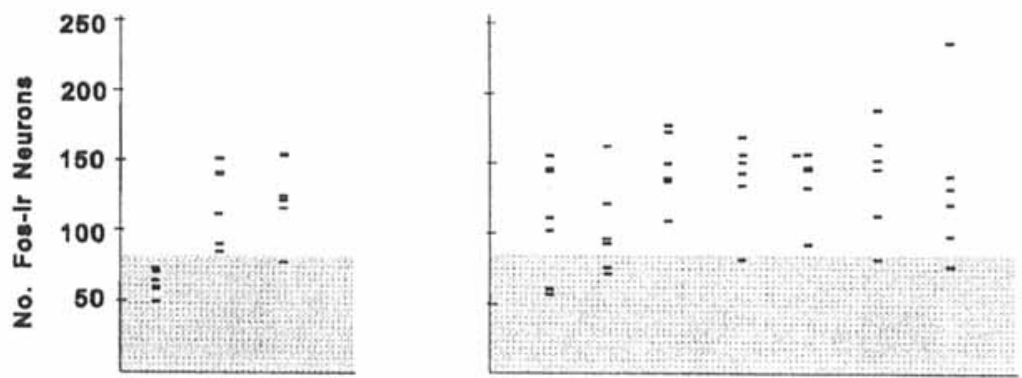

\section{BNSTpI}

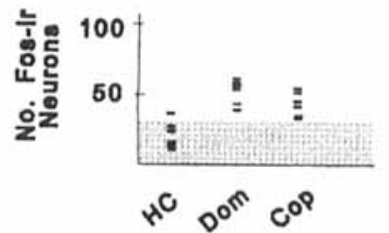

Exp. 2

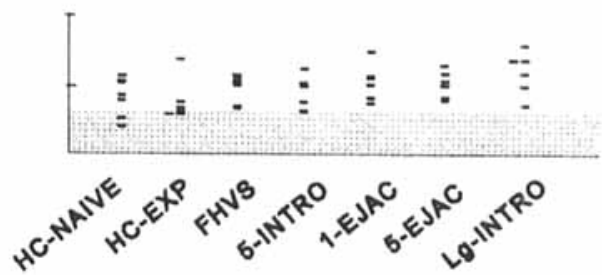

Exp. 3

FIGURE 12. Distribution of cell counts (number of Fos-ir neurons) in the anterodorsal medial amygdaloid nucleus (MeAD), the anteroventral medial nucleus (MeAV) and the posterointermediate division of the bed nucleus of the stria terminalis (BNSTpi) taken from individual male hamsters in control and experimental groups in Experiments 2 and 3, showing the increase in number and variance in the control groups in Experiment 3 (HC-NAIVE and HC-EXP) compared to the control group in Experiment $2(\mathrm{HC})$. 


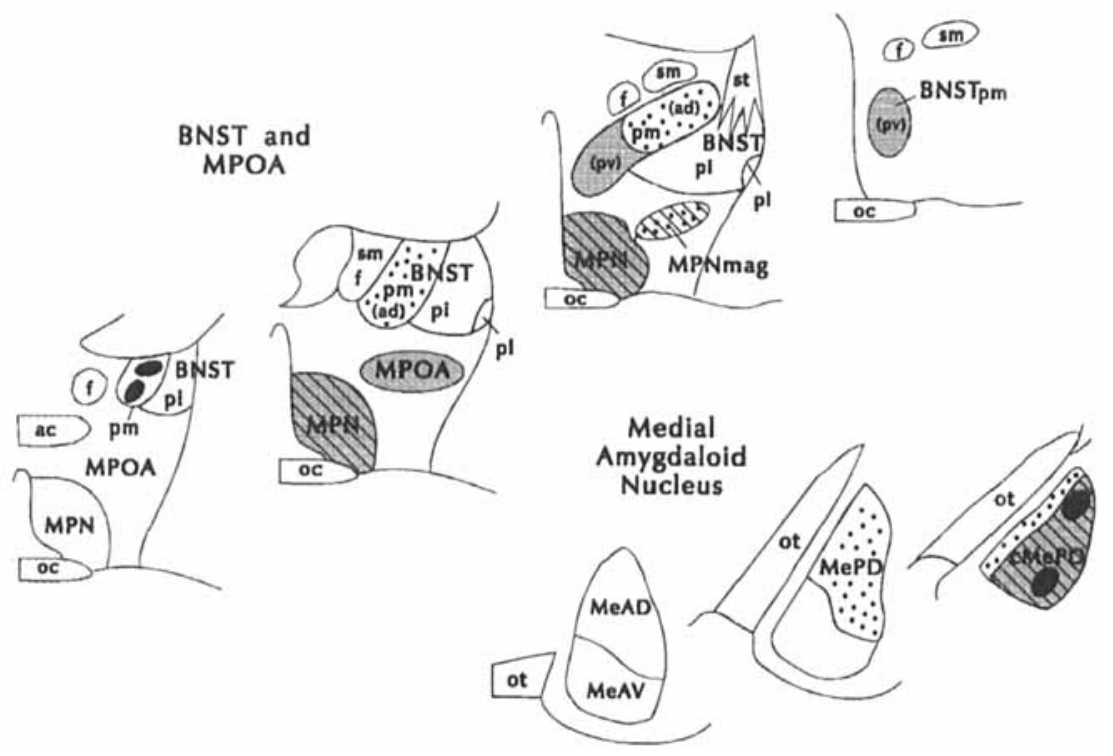

FIGURE 13. Interpretation of the results of Experiment 3, suggesting that specific subdivisions of the amygdala, bed nucleus of the stria terminalis, and medial preoptic area are selectively activated by sexual conditioning (diagonal cross-hatching), exposure to female hamster vaginal secretion FHVS (stippling), ejaculations (gray), and sexual satiety (black). See TABLE 1 for abbreviations.

Clearly, Fos-ir resulting from male sexual activity does not define function for the Fos-containing neurons; rather it identifies a neuroanatomical substrate with which to work, a substrate within which many challenges remain to determine experimentally the specific antecedents and results of neuronal activation.

With these caveats in mind, FIGURE 13 presents our interpretation of the results of studies just presented in which prior sexual experience, pheromonal stimulation, or the sequence of mating was associated with activation of individual cell groups within subdivisions of the medial amygdaloid nucleus, bed nucleus of the stria terminalis, and medial preoptic area. With the exception of the dorsolateral MPOA and the MPNmag, the areas illustrated in FIGURE 13 belong to a neuroanatomically definable network ${ }^{23}$ characterized by dense populations of sex steroid-concentrating cells that supply essential hormonal signals for mating in this species. ${ }^{30}$

Some of the cell groups in this network show gradual increases in Fos-immunoreactivity as mating proceeds, suggesting either continual recruitment of neurons in a functionally homogeneous population or sequential activation of separate functional populations. Other areas, however, appear to be specialized in function within the context of mating, such as the cell clusters in CMePD and the BNSTpm[ad] that are activated at the onset of sexual satiety. Although lesion studies did not reveal these areas to be critical for the initiation of mating, Fos-ir evidence for their role in the sequencing and termination of a copulatory bout suggests that they may be essential for the success of mating, conception. 
TABLE 1. Neuroanatomical Abbreviations

\begin{tabular}{|c|c|}
\hline ac & anterior commissure \\
\hline Aco & anterior cortical nucleus of the amygdala \\
\hline AH & anterior hypothalamic nucleus \\
\hline AHA & amygdalohippocampal area \\
\hline $\mathrm{BLa}$ & basolateral nucleus of the amygdala, anterior \\
\hline $\mathrm{BM}$ & basomedial nucleus of the amygdala \\
\hline BNST & bed nucleus of the stria terminalis \\
\hline BNSTam & BNST, anteromedial \\
\hline BNSTal & BNST, anterolateral \\
\hline BNSTpi & BNST, posterointermediate \\
\hline BNSTpl & BNST, posterolateral \\
\hline BNSTpm & BNST, posteromedial \\
\hline BNSTpm (ad) & BNSTpm, anterodorsal \\
\hline BNSTpm (pv) & BNSTpm, posteroventral \\
\hline ce & central nucleus of the amygdala \\
\hline cMePD & MePD, caudal extension \\
\hline Endo & endopiriform nucleus \\
\hline f & fornix \\
\hline IM & intercalated mass \\
\hline $\mathrm{La}$ & lateral nucleus of the amygdala \\
\hline LSv & ventral lateral septum \\
\hline MeAD & Me, anterodorsal \\
\hline MeAV & Me, anteroventral \\
\hline MePD & $\mathrm{Me}$, posterodorsal \\
\hline MePV & Me, posteroventral \\
\hline MPOA & medial preoptic area \\
\hline MPN & medial preoptic nucleus \\
\hline cMPN & medial preoptic nucleus, caudal region \\
\hline rMPN & medial preoptic nucleus, rostral region \\
\hline MPNmag & medial preoptic nucleus, magnocellular \\
\hline oc & optic chiasm \\
\hline ot & optic tract \\
\hline PAG & periaqueductal gray \\
\hline PIR & piriform cortex \\
\hline PLCo & posterolateral nucleus of the amygdala \\
\hline PMCo & posteromedial nucleus of the amygdala \\
\hline PMV & ventral premammillary nucleus \\
\hline PVN & paraventricular nucleus of the hypothalamus \\
\hline $\mathrm{rMePD}$ & MePD, rostral \\
\hline $\mathrm{sm}$ & stria medullaris \\
\hline st & stria terminalis \\
\hline VMH & ventromedial nucleus of the hypothalamus \\
\hline
\end{tabular}




\section{REFERENCES}

1. Kollack, S. S. \& S. W. Newman. 1992. Mating behavior induces selective expression of Fos protein within the chemosensory pathways of the male Syrian hamster brain. Neurosci. Lett. 143: 223-228.

2. KollaCk-WalKer, S. S. \& S. W. Newman. 1995. Mating and agonistic behavior produce different patterns of Fos immunolabeling in the male Syrian hamster. Neuroscience 66: $721-736$.

3. Fernandez-Fewell, G. D. \& M. Meredith, 1994. c-Fos expression in vomeronasal pathways of mated or pheromone-stimulated male golden hamsters: contributions from vomeronasal sensory input and expression related to mating performance. J. Neurosci. 14: $3643-3654$.

4. BAUM, M. J. \& B. J. EveriTt. 1992. Increased expression of $\mathrm{c}$-fos in the medial preoptic area after mating in male rats: Role of afferent inputs from the medial amygdala and midbrain central tegmental field. Neuroscience 50: 627-646.

5. Robertson, G. S., J. G. Pfaus, L. J. Atkinson, H. Matsumura, A. G. Philips \& H. C. FIBIGER. 1991. Sexual behavior increases c-fos expression in the forebrain of the male rat. Brain Res. 564: 352-357.

6. BAUM, M. J. \& S. R. Wersinger. 1993. Equivalent levels of mating-induced neural cfos immunoreactivity in castrated male rats given androgen, estrogen, or no steroid replacement. Biol. Reprod. 48: 1341-1347.

7. MurPhy, M. R. \& G. E. SCHNEIDER. 1970 . Olfactory bulb removal eliminates mating behavior in the male golden hamster. Science 167: 302-304.

8. PowERS, J. B. \& S. S. Winans. 1975. Vomeronasal organ: Critical role in mediating sexual behavior of the male hamster. Science 187: 961-963.

9. Winans, S. S. \& J. B. Powers. 1977. Olfactory and vomeronasal deafferentation of male hamsters: Histological and behavioral analyses. Brain Res. 126: 325-344.

10. Devor, M. \& M. R. MURPhy. 1973. The effect of peripheral olfactory blockade on the social behavior of the male golden hamster. Behav. Biol. 9: 31-42.

11. Meredith, M. 1986. Vomeronasal organ removal before sexual experience impairs male hamster mating behavior. Physiol. Behav. 36: 737-743.

12. Devor, M. 1973. Components of mating dissociated by lateral olfactory tract transection in male hamsters. Brain Res. 64: 437-441.

13. Lehman, M. N., J. B. Powers \& S. S. WinanS. 1983. Stria terminalis lesions alter the temporal pattern of copulatory behavior in the male golden hamster. Behav. Brain Res. 8: $109-128$.

14. Lehman, M. N., S. S. Winans \& J. B. Powers. 1980. Medial nucleus of the amygdala mediates chemosensory control of male hamster sexual behavior. Science 210: 557-560.

15. LehMaN, M. N. \& S. S. Winans. 1982. Vomeronasal and olfactory pathways to the amygdala controlling male hamster sexual behavior: Autoradiographic and behavioral analyses. Brain Res. 240: 27-41.

16. Kevetter, G. A. \& S. S. WinANs. 1981. Efferents of the corticomedial amygdala in the golden hamster. I. Efferents of the 'vomeronasal amygdala.' J. Comp. Neurol. 197: $81-98$

17. Lehman, M. N. \& S. S. Winans. 1983. Evidence for a ventral non-strial pathway from the amygdala to the bed nucleus of the stria terminalis in the male golden hamster. Brain Res. 268: 139-146.

18. LEHMAN, M. N. 1982. Neural pathways of the vomeronasal and olfactory systems controlling sexual behavior in the male golden hamster. Unpublished Ph.D. dissertation, University of Michigan.

19. Powers, J. B., S. W. Newman \& M. L. Bergondy. 1987. MPOA and BNST lesions in male Syrian hamsters: Differential effects on copulation and chemoinvestigatory behaviors. Behav. Brain Res. 23: 181-195.

20. MURPHY, M. R. 1976. Olfactory stimulation and olfactory bulb removal: Effects on territorial aggression in male Syrian golden hamsters. Brain Res. 113: 95-110. 
21. Bunnell, B. N., F. J. Sodetz, JR. \& D. I. Shalloway. 1970. Amygdaloid lesions and social behavior in the golden hamster. Physiol. Behav. 5: 153-161.

22. Maragos, W. F., S. W. Newman, M. N. Lehman \& J. B. Powers. 1989. Neurons of origin and fiber trajectory of amygdalofugal projections to the medial preoptic area in Syrian hamsters. J. Comp. Neurol. 280: 59-71.

23. Gomez, D. M. \& S. W. Newman. 1992. Differential projections of the anterior and posterior regions of the medial amygdaloid nucleus in the Syrian hamster. J. Comp. Neurol. 317: 195-218.

24. Canteras, N. S., R. B. Simerly \& L. W. Swanson. 1995. Organization of projections from the medial nucleus of the amygdala: A PHAL study in the rat. J. Comp. Neurol. 360: 213-245.

25. Wood, R. I., R. K. Brabec, J. M. Swann \& S. W. Newman. 1992. Androgen and estrogen receptor-containing neurons in the chemosensory pathways of the male Syrian hamster brain. Brain Res. 596: 89-98.

26. WOOD, R. I. \& S. W. NEWMAN. 1993. Mating activates androgen-receptor containing neurons in chemosensory pathways of the male Syrian hamster brain. Brain Res. 614: 65-77.

27. WoOD, R. I. \& S. W. Newman. 1995. Androgen and estrogen receptors coexist within individual neurons in the brain of the Syrian hamster. Neuroendocrinology 62: 487-497.

28. Doherty, P. L. \& P. J. Sheridan. 1981. Uptake and retention of androgen in neurons in the brain of the golden hamster. Brain Res. 219: 327-334.

29. Krieger, M. S., J. I. Morrell \& D. W. Pfaff. 1976. Autoradiographic localization of estradiol-concentrating cells in the female hamster brain. Neuroendocrinology 22: 193-205.

30. Wood, R. I. \& S. W. Newman. 1995. The medial amygdaloid nucleus and medial preoptic area mediate steroidal control of sexual behavior in the male Syrian hamster. Horm. Behav, 29: 338-353.

31. Beach, F. A. \& R. G. Rabedeau. 1959. Sexual exhaustion and recovery in the male hamster. J. Comp. Physiol. Psychol. 52: 56-61.

32. Bunnell, B. N., B. D. Boland \& D. A. Dewsbury. 1977. Copulatory behavior of golden hamsters (Mesocricetus auratus). Behaviour 61: 180-206.

33. HeEB, M. M. \& P. YAHR. 1996. c-Fos immunoreactivity in the sexually dimorphic area of the hypothalamus and related brain regions of male gerbils after exposure to sexrelated stimuli or performance of specific sexual behaviors. Neuroscience 72: 10491071.

34. Macrides, F., P. A. Johnson \& S. P. Schneider. 1977. Responses of the male golden hamster to vaginal secretion and dimethyl sulfide: Attraction versus sexual behavior. Behav. Biol. 20: 377-386.

35. MURPHY, M. R. 1973. Effects of female hamster vaginal discharge on the behavior of male hamsters. Behav. Biol. 9: 367-375.

36. Kamel, F. \& A. I. Frankel. 1978. Hormone release during mating in the male rat: Time course, relation to sexual behavior, and interaction with handling procedures. Endocrinology 103: 2172-2179.

37. Graham, J. M. \& C. Desjardins. 1980. Classical conditioning: Induction of luteinizing hormone and testosterone secretion in anticipation of sexual activity. Science 210: 1039-1041.

38. Pfeiffer, C. A. \& R. E. Johnston. 1994. Hormonal and behavioral responses of male hamsters to females and female odors: Roles of olfaction, the vomeronasal system, and sexual experience. Physiol. Behav. 55: 129-138.

39. Fiber, J. M., P. Adames \& J. M. Swann. 1993. Pheromones induce c-fos in limbic areas regulating male hamster mating behavior. Neuroreport 4: 871-874.

40. COOLEN, L. M. 1995. The neural organization of sexual behavior in the male rat: A functional neuroanatomical Fos-study. Unpublished Ph.D. dissertation. University of Nijmegen. 
41. Huck, U. W. \& R. D. Lisk. 1985. Determinants of mating success in the golden hamster (Mesocricetus auratus): I. male capacity. J. Comp. Psychol. 99: 98-107.

42. Kollack-Walker, S. S. \& S. W. Newman. 1996. Mating-induced expression of c-fos in the male Syrian hamster brain: Role of experience, pheromones and ejaculations. $J$. Neurobiol., in press.

43. LanieR, D. L., D. Q. Estep \& D. A. Dewsbury. 1975. Copulatory behavior of golden hamsters: Effects on pregnancy. Physiol. Behav. 15: 209-212.

44. HucK, U. W. \& R. D. LisK. 1985. Determinants of mating success in the golden hamster (Mesocricetus auratus): II. pregnancy initiation. J. Comp. Psychol. 99: 231-239. 